Article

\title{
Important Factors for Improving Google Search Rank
}

\author{
Christos Ziakis * (1), Maro Vlachopoulou, Theodosios Kyrkoudis and Makrina Karagkiozidou
}

ISEB lab, Dep. of Applied Informatics, University of Macedonia, 156 Egnatia St., 54006 Thessaloniki, Greece; mavla@uom.edu.gr (M.V.); it1421@uom.edu.gr (T.K.); it1414@uom.edu.gr (M.K.)

* Correspondence: ziakis@uom.edu.gr; Tel.: +30-693-746-2999

Received: 11 December 2018; Accepted: 18 January 2019; Published: 30 January 2019

\begin{abstract}
The World Wide Web has become an essential modern tool for people's daily routine. The fact that it is a convenient means for communication and information search has made it extremely popular. This fact led companies to start using online advertising by creating corporate websites. With the rapid increase in the number of websites, search engines had to come up with a solution of algorithms and programs to qualify the results of a search and provide the users with relevant content to their search. On the other side, developers, in pursuit of the highest rankings in the search engine result pages (SERPs), began to study and observe how search engines work and which factors contribute to higher rankings. The knowledge that has been extracted constituted the base for the creation of the profession of Search Engine Optimization (SEO). This paper consists of two parts. The first part aims to perform a literature review of the factors that affect the ranking of websites in the SERPs and to highlight the top factors that contribute to better ranking. To achieve this goal, a collection and analysis of academic papers was conducted. According to our research, 24 website characteristics came up as factors affecting any website's ranking, with the most references mentioning quality and quantity of backlinks, social media support, keyword in title tag, website structure, website size, loading time, domain age, and keyword density. The second part consists of our research which was conducted manually using the phrases "hotel Athens", "email marketing", and "casual shoes". For each one of these keywords, the first 15 Google results were examined considering the factors found in the literature review. For the measurement of the significance of each factor, the Spearman correlation was calculated and every factor was compared with the ranking of the results individually. The findings of the research showed us that the top factors that contribute to higher rankings are the existence of website SSL certificate as well as keyword in URL, the quantity of backlinks pointing to a website, the text length, and the domain age, which is not perfectly aligned with what the literature review showed us.
\end{abstract}

Keywords: SEO; search engine optimization; website ranking; ranking factors

\section{Introduction}

The emergence of the Internet, and its rapid expansion worldwide, resulted in the storage and sharing of a massive amount of data, and this data was available to every user with an internet connection. As a result, billions of websites were created, which made it hard for the average user to extract useful information from the web efficiently for a specific search. The need for an easier, more efficient way to search for information led to the development of search engines. Gradually, search engines began to assess the relevance of every website on their indexes compared to the queries provided to them by the users. They took into consideration several website characteristics and metrics and calculated the value of each website using complex algorithms. The enormous number of websites being indexed from search engines, along with the increasing competition for the first search results, led to studying and implementing various techniques in order for websites to appear 
more valuable in search engines. These techniques make up what is called today "Search Engine Optimization" (SEO) and they are divided into either black hat or white hat depending on whether they violate the search engines' terms of service. It is worth mentioning that a website nowadays has to be indexed into the first page of SERPs in order to receive a sufficient amount of organic visitors. Ideally one of the first three results has to be acquired for the targeted keywords as the click through rates are $30 \%$ (1st position), 16\% (2nd position), and 10\% (3rd position), while the click through rate for positions past the first page of SERPs is limited to under $2 \%$ [1]. Search engines nowadays change their algorithms regularly by adding and removing factors affecting the ranking of websites: SEO is a dynamic procedure.

In this paper, a literature review, as well as a research review was conducted in order to pinpoint the main factors that affect the ranking of a website in the search engines' results. The knowledge from this paper aims to provide enterprises and freelancers with a guide on the best techniques to maximize a website's position in search results.

\section{Methodology}

In order to accomplish the study, we researched several scientific databases to find articles about SEO. The keyword used in our research is "Search Engine Optimization".

To choose the most relevant articles, we searched academic papers using the PRISMA methodology [2] as shown on Figure 1 on the digital libraries of (i) Science Direct, (ii) Springer, and (iii) IEEE Xplore during February and March of 2018. The first search resulted in almost 1010 citations. By applying the PRISMA methodology we retrieved 125 articles after title and abstract screening and used 23 articles as the base for the literature review.

The fact that the papers did not include some crucial factors that affect the ranking of a website lead us to search around the web, using the same keywords, for authorized websites including the factors that were not included in academic papers. Information only from trustworthy websites was extracted only after the opinions of the authors were carefully examined.

Concerning the research, we implemented an 8-step process to get the requested findings

1. We searched for primary research to highlight the top SEO factors.

2. We determined the factors that we will use in our research and the tools that will be used to extract data for every factor.

3. We determined the number of the samples, the size of every sample and the keywords that are going to be used for every sample.

4. A Google search was conducted using the keywords of every sample and the data was saved in EXCEL spreadsheets.

5. We normalized the data using a common numeric scale.

6. We conducted a statistical analysis using the Spearman correlation coefficient.

7. The average of the samples' Spearman correlation coefficients for every factor was calculated.

8. We represented the SEO factors graphically according to the significance of every factor.

More specifically, the research was conducted using three samples from which the first 15 search results were extracted. The key words that we used were "Hotel Athens", "email marketing", and "casual shoes". The first search was conducted on April 1, 2018 at 11:45 pm, the second on April 12, 2018 at 2:40 pm, and the third on April 13, 2018 at 4:00 pm. We chose Google as the search engine of the study as it is the most popular search engine with an almost $90 \%$ market share [3].

Concerning the factors that we took in consideration for the study, instead of using domain or page authority metrics we used the number of backlinks as a metric of the websites' value following the methods of former SEO studies [4-6].

The normalization of the data was made using as a base a numeric scale from 0 to 9 in order to compare the samples directly. We replaced the missing values with the average of the rest normalized data and we consider that it does not affect the outcome of the research. 
We analyzed the data using the Spearman correlation coefficient. We used this method of statistical analysis because we are not sure that there is linear correlation between every SEO factor and the ranking of each website in the search results, [7] trying to prove the existence of monotony between them. However, we have to emphasize that correlation does not mean always causation, so we can make assumptions safely only for the factors that are highly correlated $(|r|>0.5)$ to the ranking of the websites. The values of the correlations are calculated with the web tool vassarstats.net [8] and for the measurement of the correlation degree we used the following scale.

$$
\begin{gathered}
\mathrm{R} \leq-0.5 \text { strong correlation } \\
-0.5<\mathrm{r} \leq-0.3 \text { medium correlation } \\
-0.3 \leq \mathrm{r}<-0.1 \text { weak correlation }
\end{gathered}
$$

We use the negative scale to assess whether a factor correlates to high ranking in the search results as higher score means numerically lower ranking position (from 15th to 1st position).

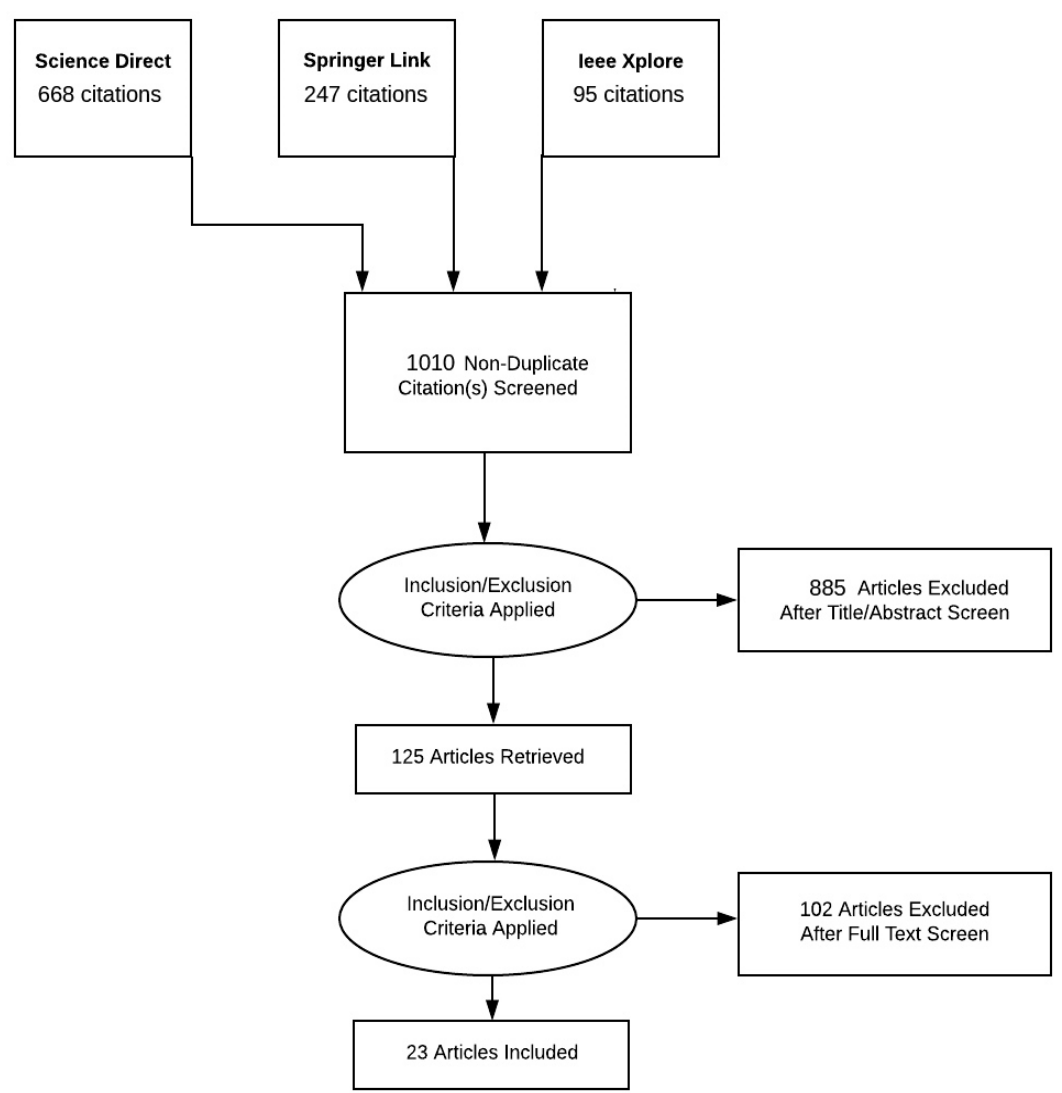

Figure 1. Application of PRISMA methodology.

\section{Literature Review}

We conducted a literature review to address the main factors that affect the ranking of a website as mentioned in former papers. Yalçın et al. (2010) [9], beyond the report of some factors that affect SEO, illustrated the mechanics behind the operation of the search engines. They concluded that SEO is a dynamic process which must be monitored frequently, tracing positive or negative changes for the improvement of a website. Wilson et al. (2006) [10] discussed particular SEO techniques that should be implemented during the creation of a website that aim to make the website visible on search engines. In their opinion, keywords must be related to the content of the website so it can be indexed higher in the search results for the targeted keywords. Finally, it is stressed that SEO is a 
competitive sector and changes are made often. Similar topics are analyzed by Zilincan (2015) [11] and Cui et al. (2011) [12]. Rehman et al. (2013) [13] evaluated the existing SEO techniques by analyzing and comparing other researches while mentioning main points that could be optimized. Using these data, they recommend some theoretical methods for SEO. This is also the main topic of the research of Zhang et al. (2011) [14] who conducted a comparative research on the SEO factors using 116 websites. Killoran (2013) [15] studies the factors that affect the ranking of the SERPs and how web developers and marketers can take advantage of them. He mentions that SERPs are shaped by three categories of participants: the search engine companies and programmers, the SEO experts, and the search engine users. It is claimed that not only the target group of the website but also the competition has to be considered while choosing keywords. Finally, he recommends that correct keyword placement and link-building through interaction with other content creators are crucial for optimal results. The same pattern has been followed by Thekral et al. (2016) [16] who focused on the theoretical documentation of the above aspects. Kakkar et al. (2015) [17] compared and explained various algorithms of Google and focuses on SEO strategies and how they lead to better rankings, concluding that SEO is a long term and dynamic procedure. Gudivada et al. (2015) [18] analyzed the mechanics behind search engines and the techniques that are used to rank websites as well as how much it impacts the traffic of a website. They conducted a study which shows that $70 \%$ of users prefer the organic results. More specific, most of them (60\%) select one of the first three organic results. That proves the impact of SEO on website promotion. Krrabaj et al. (2017) [19] researched the on-site as well as the off-site SEO factors. According to the results, quality content and inbound and outbound links affect mostly the ranking of a website in the search results. Accordingly, Evans (2007) [20] made a research on the most popular SEO techniques that affect the ranking of websites in the SERPs. The sample of the research included 50 SEO optimized and 50 nonoptimized websites and the research revealed the most effective SEO techniques as well as the importance of backlinks for a successful SEO campaign. Chen et al. (2011) [21] aim to combine the SEO knowledge with the tools that the world wide web offers in order to show how to increase the traffic of a website and provide a better relationship with the website visitor. Patil Swati et al. (2013) [22] studied the ranking algorithms of the search engines and presented the main factors that affect the SEO of a website. They categorized them in "white hat" and "black hat" too, while pinpointing the differences between them. Hui et al. (2012) [23] referred analytically to the mechanics behind the operation of search engines as well as the factors that affect the ranking of websites and the method they used to select the search engine for their study. Gregurec et al. (2012) [24] briefly presented the SEO factors that have been mentioned in previous papers and they analyze some Croatian websites on the topics of computer science and engineering. They concluded that independently from the country that a website serves the same principles of SEO are applied. Chandra et al. (2014) [25] showed 32 efficient and effective methods of detecting spam in the URL, the content and the links of a website. To accomplish this, they created a classifier that is based on neural networks and requires minimal processing power. Thakur et al. (2011) [26] aim to simplify the steps for the optimization of websites to make the process easier for marketing experts. They present several SEO techniques mentioning the most flexible and effective of them. Their research constitutes a guideline for marketers in order to adopt the best techniques. Ergi et al. (2014) [27] found out in their research that the loading speed, the low bounce rate, and the total traffic of a website have a positive impact on the ranking of a website. Kumar et al. (2011) [28] study the characteristics of the algorithms that the search engines use and they suggest an optimization strategy for websites. The application of the strategy was proved effective and gave information for even better ways of website optimization. Dean (2016) [29] made a primal SEO research with the use of a crawler which crawled 1 million websites, and information about them was gathered. The main purpose of the research was to compare the ranking of the websites in the SERPs and the SEO techniques used on them. He concluded that backlinks affect mostly the position of a website followed by relevant content, the existence of at least one image as well as the existence of SSL certificate. However, they found out that techniques such as the existence of the preferred keyword in the title tag do not affect the SEO 
of a website. Fishkin (2017) [30], in his article, included the main factors that will affect a website's position in 2018. He provides marketers a checklist with all the data that they should take into consideration according SEO. He suggests that marketers should focus on providing value through their content while optimizing the website's structure in order to provide better user experience and content traceability. Finally, Palos-Sanchez et al. (2018) [31] focused on analyzing user behavior when using Internet applications. They found out that technology companies should stop recommending undifferentiated strategies and to adopt processes to emphasize on better user experience in a website.

\section{Findings}

\subsection{Literature Review}

Through the literature review we found that the factors as shown on Table 1 impact the ranking of websites in search engine indexes. On Table 2 the matching of previous researches to indexes is presented.

Table 1. Previous research on search engine optimization (SEO) factors.

\begin{tabular}{cc}
\hline Page Size and Website Loading Time & {$[7,11,21,24,26,30]$} \\
Keyword in Title tag & {$[8,10,11,19,20,23,25,27,30]$} \\
Keyword in H1/H2/H3 Tag & {$[8,19,20,30]$} \\
Keyword Density in Text & {$[7,8,11,19,20,25,30]$} \\
Keyword in URL & {$[8,19,20,30]$} \\
Keyword in Meta Description Tag & {$[8,15,19,20,25,30]$} \\
Alt Text & {$[10,13,15,23]$} \\
Unique - High Quality Content & {$[10,13,14,20,30]$} \\
Title Length/ Description Length & {$[7,9,11,19,22]$} \\
URL Length & {$[8,9,13,19,21,22,26,30]$} \\
Text Length & {$[22,26]$} \\
Text to Code Ratio & {$[22]$} \\
Internal Linking & {$[17,20,25]$} \\
Website Structure & {$[7,9,14,16,19,25]$} \\
Social Media Support & {$[7,9,12-16,18,23,30]$} \\
Custom 404 page & {$[11,15,21]$} \\
SSL Certificate & {$[22,26,30]$} \\
Sitemap xml file & {$[7,8,11,15,21]$} \\
Domain age & {$[7-9,11,17,25]$} \\
Responsive layout & {$[14,15,20]$} \\
W3C Validity & {$[8]$} \\
Bounce Rate & {$[24-26]$} \\
Time on Site & {$[24]$} \\
\hline
\end{tabular}

The most mentioned factors in the above papers include the quality and quantity of backlinks, the social media support, the keyword in title tag, the URL length, and the website structure.

Page size and website loading time: A very important on-page factor for the optimization of a website is the loading time. Search engines include this factor in their algorithms too. The higher the loading time, the lower the ranking of a website in the search results. Although special effects and graphic elements upgrade the image of a website, the excessive use of them may increase the loading time of the website. Loading speed is affected not only by graphic elements but the HTML file and all the elements regardless of their file type contribute to the size of a website and affect the loading speed. Most search engines will not fully index pages that are greater than a certain sizeKeyword in title tag: The title tag refers to the title of the file. Every HTML/XHTML file contains a title element. The main use of the title tag is to determine the title in the toolbar of the browser, it shows the title of the website in the search results and the name in the 'favorites' tab. Concerning this factor while structuring a website it is easier for the search engines to trace and rank it in their search results. 
Keyword in $\mathrm{h} 1 / \mathrm{h} 2 / \mathrm{h} 3$ tag: Equally important on page intervention is the use of keywords in $\mathrm{h} 1, \mathrm{~h} 2$, and $\mathrm{h} 3$ tags. This element is the second most important on page factor for higher ranking in the search results. The websites with optimized and targeted $h 1, h 2$, and $h 3$ tags are ranked higher by the search engines. This happens as the crawler searches for information regarding the content structure of every website and the best way to extract this piece of information is to search on these tags. Skipping this part of optimization is a big obstacle on the way to higher ranking.

Keyword density in text: The keyword density is the number of times the targeted keyword appears in the text of a website compared to the rest text. This on-page factor and the frequency of times the keyword appears in title tag are considered some of the most important factors when optimizing a website. The ideal keyword density range is 2 to $8 \%$ of the text of the page but this range is not universally true and is affected by other factors. We have to pinpoint that the keyword density should not have negative impact on the readability of the text because it is perceived as negative element of a website by the search engines

Keyword in URL: It is easier for crawlers to trace a website if its URL contains the targeted keyword. In general, search engines tend to rank higher websites with .edu and .gov domains as these domains are used by state and educational websites. However, optimization for all types of websites can be achieved if the length of the URL is kept short.

Keyword in meta description tag: The meta description tag is a summary of the content of a webpage. This tag contains the text that appears in the search results of the search engines just below the link. The meta description tag is a guide on what keywords should the website be indexed for by the search engines

Alt text: To be ensured that the most elements of a website are indexed correctly, they have to be in HTML format. However, content such as images and videos need more information to be indexed by the search engines. Webmasters must use an alt tag for images and a transcript for videos to provide enough details about those types of content.

Unique high-quality content: Search engines prefer websites with unique, authentic, and quality content. If the content of a website is plagiarized from another website it will not be ranked high by the search engines. In addition, a frequently updated content has better chance to get ranked higher in search engines for related keywords.

Title length \& Description length: The title of the website must reflect the topic of the website without unnecessary information. With the use of a short and comprehensive title, website visitors can understand the main topic of the website. Google suggests no more than 70 characters for title tag. The meta tag description offers some pieces of information in the search engines about the website. A poor or no description in this tag suggests a low-quality website. On the other side, larger than standard text (up to 155 characters recommended by Google) may be considered spam as the targeted keywords may be used excessively.

URL length: The URL represents the address of the site in the World Wide Web. As mentioned above, the targeted keywords should be included in the URL, so it can be traced easily from the search engines. To become even more SEO-friendly it should be short and understandable.

Text length: Websites with longer text tend to rank higher in the search results compared to the ones that have less or no text at all. This proves that search engines prefer content rich websites. This assumption may correlate with the fact that in richer content the targeted keywords appear more frequently. Besides, users prefer longer text as it is more informative. Text to code ratio: The text-to-code ratio is a metric that represents the ratio between the text in the front-end part of the site to the back-end code. The ideal ratio range is 25 to $70 \%$. This ratio refers in the visual text compared with all the HTML elements including the image tags and other visual elements. Although this factor is not directly correlated to the ranking of a website, there are many factors that are based on this factor, so it is crucial to be considered for a more effective SEO strategy.

Internal linking: A very important SEO factor is the linking of the individual pages of a website. The goal of internal linking is not only to make the visit of the user more enjoyable but also to facilitate 
the tracing and the indexing of the individual components of the website. As a result, a higher ranking of the website is achieved, given the fact that the value that is passed by the external links is shared uniformly in all subpages of the website. Finally, it should be mentioned that internal links must be checked regularly to trace any broken links.

Quality and quantity of backlinks: The quality and quantity of backlinks is one of the most important factors for optimal ranking. Backlinks are references from other websites pointing to the targeted website. Google, in order to determine the importance of each website for the user, invented an algorithm that calculates the value of the website based on the references from other websites pointing to the given site. The algorithm takes into consideration the number as well as the quality of the incoming links. This algorithm is called "Pagerank" and uses a 0 to 10 scale to determine a relative score of that page's importance and authority. The ranking of a website heavily relies on the Pagerank algorithm, which is the only factor that has remained unchanged despite the updates made in the ranking patterns. Considering the importance of this factor, building quality backlinks that are referenced from a high number of websites is extremely important to achieve high ranking. Depending on this, webmasters started implementing black hat SEO techniques such as purchasing links, arranging for links provided by link farms, and link exchange with other websites to quickly build inbound links. As soon as these techniques became known to search engine programmers, websites using these link-building strategies were banned and the algorithms were changed in order to value quality over quantity. Therefore, when building backlinks, the authority of the linking sources and the topic relevance of the linked sites must be considered.

Website structure: Having a clear navigation system is a standard requirement for a useful website. Some websites use frames and navigation buttons made in java or other programming languages which are not traceable by search engines. The solution to this problem is the creation of a complementary navigation bar using normal HTML links to ensure that every page of the website is traced by search engines. This type of navigation bar helps crawlers extract more information about the structure of the website than graphic elements. The structure of the website has to be clear as mentioned above in order to minimize the directory depth. Ideally the structure consists of less than four layers. This type of structure is achieved by embedding the most important pages, links, and titles from the second and third layer into the home page.

Social media support: It is widely known that most people use social media daily and it is the only source of information for some of them. Connecting a website to social media accounts ensures that the website gains more traffic as well as quality backlinks which increase its authority. Based on these facts, search engine algorithms begun to depend increasingly on bookmarks, social signals, and the impact of content creators on social media to assess the value of a website.

Custom 404 page: Web Servers return a 404 webpage when the requested webpage cannot be found. Customizing the 404 page helps keep users on the site and can even enhance their search experience. The customization includes pointers to home page or other pages of the site and even references to other sites with relevant to the user's search content.

SSL certificate: The acronym SSL refers to the term secure socket layer. It is a protocol that establishes an encoded link between the server and the browser that allows sensitive information to be transmitted securely. The SSL certificate ensures that a website is reliable and that the user's data are kept safe. In addition, it prevents spamming. The websites that have this certificate installed use the protocol https instead of http. Such websites tend to rank higher in the search results as they are safer than similar sites without the certification.

Sitemap xml file: An XML file that is created by the web developer of the website and is submitted for use by search engines. The creation and the uploading of a sitemap along with the website help the crawler to find all the subpages of a website. It also notifies the search engines for any changes made to the website, the degree of significance of every page, the frequency of the website updates, and other information. In sum, it contains information that enhances the effectiveness and the relevance of the indexed website's content but also makes it easier for search engines to index the 
website. This procedure is crucial for the optimization of a website as the number of a website's pages is highly correlated to the website's ranking.

Domain age: The age of a domain name is quite an important factor affecting the ranking of a website. According to Google's algorithms, the older the domain the more reliable the website that the domain points to. It is no surprise that websites with newer domains tend to rank lower that those with older ones.

Responsive layout: According to a 2014 study on the subject "Mobile path to Purchase" by Telemetrics and xAd [15], 50\% of the respondents use a mobile device to start their search and two out of three buyers purchase goods and services through their research. Even though there is clear evidence that the mobile devices contribute to e-commerce success, many websites are not responsive to mobile devices. A responsive website not only enhances the experience of the user but also contributes to higher rankings. When making a website responsive, services that mobile devices introduced such as voice search must be considered. It is important to optimize the keywords in order to allow search using whole sentences instead of keywords.

W3C validation: W3C or the World Wide Web consortium is a worldwide web commission. This commission sets some standards regarding syntax errors in the source code of the website. Every website that meets the standards that the consortium sets ranks higher in the search engines. Websites which are validated can add a small icon that indicates that they are w3c validated.

Bounce rate: The metric that shows the percentage of visitors that leave the website just after they view the first page of the website without exploring other pages. Low bounce rate indicates a website with high quality content which is also relevant to the user's search. That is why search engines recently included that metric as a factor that affects the ranking of a website in the search results.

Time on site: The total duration of a user's visit in a website. It is obvious that the more time a user spends on a website the more valuable the content of the website is for the search engines as it is more likely for a visitor to spend more time on a page with valuable for them content. Considering the usefulness of this metric, it is becoming an increasingly more important indicator for the ranking of a website.

\subsection{Our Research}

After the analysis of the data for every website of the three samples that we gathered, we calculated the Spearman correlation between ranking of each website and their scores based in our scales, and then we calculated the averages for each factor and presented them concentrated using a table.

Table 2 presents all the correlations as they emerged from the samples. The columns present the samples and the rows present factors. The last row presents the average of the correlations for every factor. The averages show whether a factor is universally valid. The results are presented on Figure 2 in descending order.

We should mention that for the factors with low correlation and high score we cannot be sure whether they contribute to the ranking of websites. It is possible that the constant high score is correlated to higher ranking but we cannot confirm that as the sample of search results for every keyword is restricted to 15 webpages.

Table 2. Averages for each factor.

\begin{tabular}{ccccc}
\hline Seo Factors & Hotel Athens & Email Marketing & Casual Shoes & Average \\
\hline $\begin{array}{c}\text { Keyword in Title } \\
\text { Tag }\end{array}$ & 0.0511 & 0.0000 & 0.2456 & 0.0989 \\
Keyword in & -0.5404 & -0.2359 & 0.2107 & -0.1885 \\
H1/H2/H3 Tag & -0.0677 & 0.3049 & -0.2364 & 0.0003 \\
Keyword Density & -0.5890 & -0.1362 & -0.2443 & -0.3232 \\
Keyword in URL & & & & \\
\hline
\end{tabular}


Table 2. Cont.

\begin{tabular}{ccccc}
\hline Seo Factors & Hotel Athens & Email Marketing & Casual Shoes & Average \\
\hline Keyword in meta & -0.0323 & -0.0691 & 0.1809 & 0.0265 \\
description Tag & 0.2270 & -0.1745 & 0.1237 & 0.0587 \\
Alt text & 0.0631 & -0.3293 & 0.0000 & -0.0887 \\
Title Length & 0.4373 & 0.4908 & -0.2716 & 0.2188 \\
URL Length & -0.0094 & -0.2253 & 0.0619 & -0.0576 \\
Text to Code ratio & -0.4582 & -0.2330 & -0.1727 & -0.2880 \\
Text Length & -0.6771 & -0.2914 & 0.0686 & -0.3000 \\
Quantity of & 0.1745 & 0.0000 & 0.2474 & 0.1406 \\
Backlinks & -0.3928 & -0.3093 & -0.3093 & -0.3371 \\
404 Page & -0.1260 & 0.2270 & 0 & 0.0337 \\
SSL Certificate & -0.5652 & 0.0229 & -0.1659 & -0.2361 \\
Sitemap XML & -0.4779 & -0.1130 & -0.2535 & -0.2815 \\
Number of Site & 0.2474 & 0.0908 & 0.0000 & 0.1127 \\
$\quad$ Pages & 0.3712 & 0.0619 & -0.3712 & 0.0206 \\
Domain Age & -0.4942 & -0.0092 & 0.1605 & -0.1143 \\
Responsive & -0.5043 & -0.0092 & 0.3499 & -0.0545 \\
$\quad$ Layout & -0.6444 & 0.4415 & 0.2623 & 0.0198 \\
W3C Validation & & & &
\end{tabular}

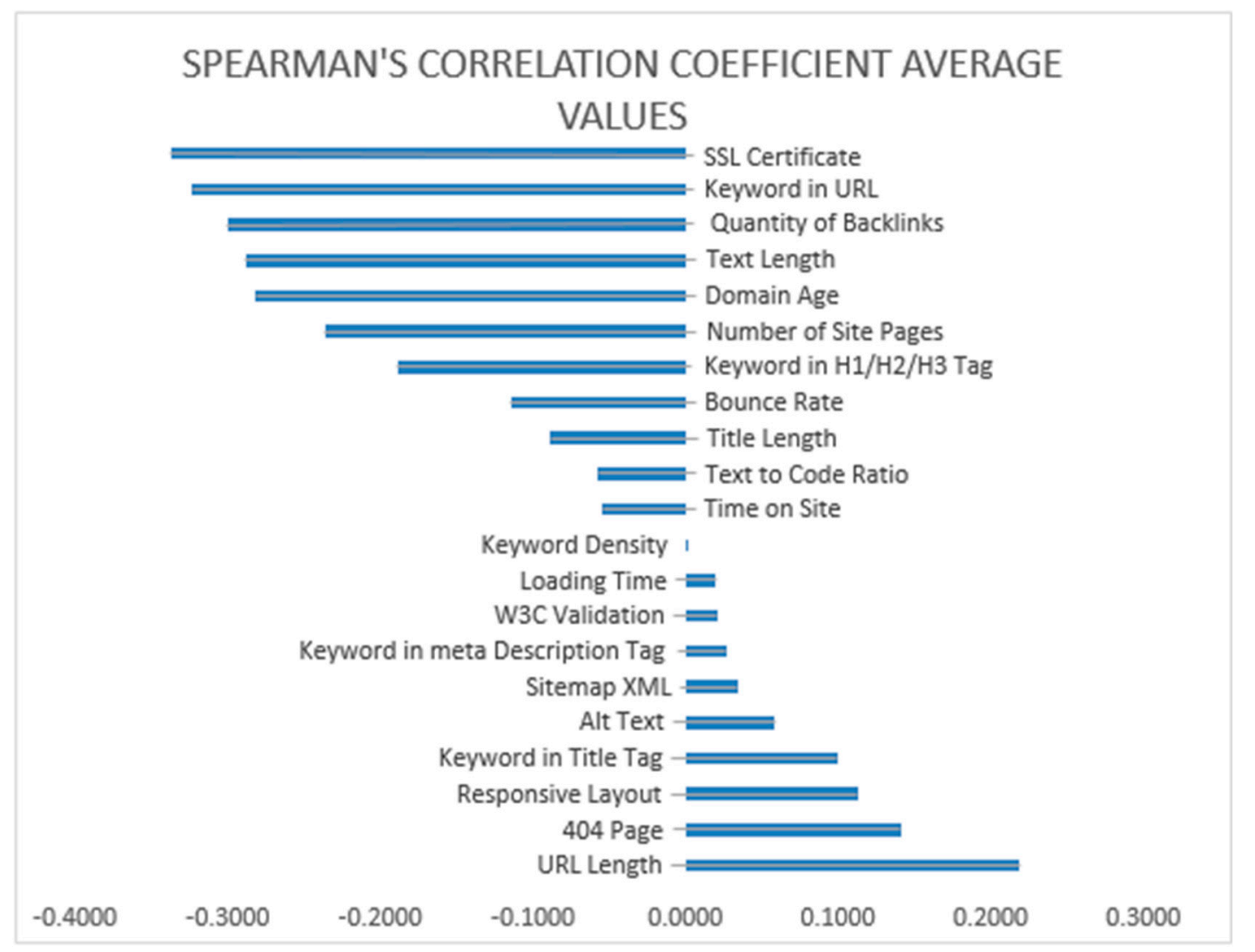

Figure 2. Spearman's correlation coefficient average values.

\subsection{Discussion}

In comparison with other studies on the topic $[26,28,29]$, we observe that the factors that are crucial despite the changes in algorithms are the quantity and quality of the backlinks and, to a lesser extent, the bounce rate and the SSL certificate. The importance of the quantity is confirmed by our 
study, but we did not have the means to check whether the quality of backlinks is important too. Contrary to the results of other studies we found out that the website loading time, the URL length, and the use of targeted keywords in the title tag do not affect the ranking of a website, although the difference in the results are due to the approach of our study compared to the others. More specifically, in Dean's study [26], the sample contained less search results for more keywords. In the other one conducted in 2015 by Moz [28], the changes in the algorithm of the search engine may have affected the factors that affect the results.

\section{Conclusions}

The competition among the websites for the SERPs is huge so a convenient optimization plan is needed: a plan that includes a holistic approach regarding the SEO factors but depends on the most effective ones. As our study revealed, some factors determine the bulk of success in term of ranking high in the SERPs and they have remained unchanged over time. However, search engine algorithms tend to change often, and new factors are added while outdated or not effective factors are excluded. This is why web developers must check the algorithm changes and adjust their websites in order to not only achieve but also maintain high ranking in search engines. This study attempts to show the most effective SEO factors for high ranking in search engines and the significance for the most important factors that affect SEO.

\subsection{Implications for Academics}

In this paper, 24 SEO factors are included with all of them being equally analyzed, providing the opportunity to explore the most important factors that affect SEO in one place. This study can be used as a base for future studies with the use of better infrastructure in order to reveal more detailed results regarding the factors that affect the ranking of a website in the search engines.

\subsection{Practical Implications}

Via this research we would like to pinpoint that the results of our study aim not to reveal the patterns and mechanics behind the algorithms of search engines, but to educate and guide the owners of small- and medium-sized businesses, as long as marketers about the most effective factors that optimize the ranking of a website in the search results of a popular search engine such as Google, so that they can be able to plan an effective optimization strategy for their websites without having to use specialized technological tools.

Author Contributions: Conceptualization, C.Z. and M.V.; Methodology, C.Z. and T.K.; Validation, T.K. and M.K.; Formal Analysis, M.K.; Investigation, T.K. and C.Z.; Resources, M.K.; Data Curation, T.K.; Writing-Original Draft Preparation, T.K.; Writing—Review \& Editing, M.K.; Visualization, T.K.; Supervision, C.Z.; Project Administration, C.Z. and M.V.

Funding: This research received no external funding.

Conflicts of Interest: The authors declare no conflicts of interest.

\section{References}

1. Google Organic CTR History. Fresh CTR Averages Pulled Monthly from Millions of Keywords. 2018. Available online: https:/ /www.advancedwebranking.com/ctrstudy/ (accessed on 25 December 2018).

2. Moher, D.; Liberati, A.; Tetzlaff, J.; Altman, D.G.; the PRISMA Group. Preferred Reporting Items for Systematic Reviews and Meta-Analyses: The PRISMA Statement. Ann. Intern. Med. 2009, 151, $264-269$. [CrossRef] [PubMed]

3. Search Engine Market Share Worldwide-December 2018. Available online: http://gs.statcounter.com/ search-engine-market-share\#monthly-201806-201806-bar (accessed on 25 December 2018).

4. Dean, B. Backlinko Ranking Factors Study: Methods \& Results. 2 September $2016 . \quad$ Available online: https://backlinko.com/wp-content/uploads/2016/02/Search_Engine_Ranking_Factors_Study_ Methods_Backlinko.pdf (accessed on 24 January 2018). 
5. Search Engine Ranking Factors 2015. 2015. Available online: https://moz.com/search-ranking-factors (accessed on 6 June 2018).

6. Ranking Factors SEMrush Study 2.0. 2017. Available online: https:/ / email.semrush.com/acton/attachment/ 13557/f-0e30/1/-/-/-/-/SEMrush_Ranking_Factors_Study_2_0.pdf (accessed on 6 June 2018).

7. Hendrickson, B. Statistics a Win for SEO. 2010. Available online: https://moz.com/blog/statistics-a-winfor-seo (accessed on 15 April 2018).

8. Spearman Rank-Order Correlation Coefficient. Available online: http://vassarstats.net/corr_rank.html (accessed on 21 April 2018).

9. Yalçın, N.; Köse, U. What is search engine optimization: SEO? Procedia Soc. Behav. Sci. 2010, 9, 487-493. [CrossRef]

10. Wilson, R.F.; Pettijohn, J.B. Search engine optimisation: A primer on keyword strategies. J. Direct Data Digit. Mark. Pract. 2006, 8, 121-133. [CrossRef]

11. Zilincan, J. Search engine optimization. In Proceedings of the CBU International Conference Proceedings, Prague, Czech Republic, 25-27 March 2015; Volume 3, p. 506.

12. Cui, M.; Hu, S. Search engine optimization research for website promotion. In Proceedings of the 2011 International Conference on Information Technology, Computer Engineering and Management Sciences (ICM), Nanjing, China, 24-25 September 2011; Volume 4, pp. 100-103.

13. Rehman, K.; Khan, M.A. The foremost guidelines for achieving higher ranking in search results through search engine optimization. Int. J. Adv. Sci. Technol. 2013, 52, 101-110.

14. Wang, F.; Li, Y.; Zhang, Y. An empirical study on the search engine optimization technique and its outcomes. In Proceedings of the 2011 2nd International Conference on Artificial Intelligence, Management Science and Electronic Commerce (AIMSEC), Deng Feng, China, 8-10 August 2011; pp. 2767-2770.

15. Killoran, J.B. How to use search engine optimization techniques to increase website visibility. IEEE Trans. Prof. Commun. 2013, 56, 50-66. [CrossRef]

16. Gupta, S.; Rakesh, N.; Thakral, A.; Chaudhary, D.K. Search engine optimization: Success factors. In Proceedings of the 2016 Fourth International Conference on Parallel, Distributed and Grid Computing (PDGC), Pradesh, India, 22-24 December 2016; pp. 17-21.

17. Kakkar, A.; Majumdar, R.; Kumar, A. Search engine optimization: A game of page ranking. In Proceedings of the 2015 2nd International Conference on Computing for Sustainable Global Development (INDIACom), New Delhi, India, 11-13 March 2015; pp. 206-210.

18. Gudivada, V.N.; Rao, D.; Paris, J. Understanding search-engine optimization. Computer 2015, 48, 43-52. [CrossRef]

19. Krrabaj, S.; Baxhaku, F.; Sadrijaj, D. Investigating search engine optimization techniques for effective ranking: A case study of an educational site. In Proceedings of the 2017 6th Mediterranean Conference on Embedded Computing (MECO), Bar, Montenegro, 11-15 June 2017; pp. 1-4.

20. Evans, M.P. Analysing Google rankings through search engine optimization data. Internet Res. 2007, 17, 21-37. [CrossRef]

21. Chen, C.Y.; Shih, B.Y.; Chen, Z.; Chen, T.H. The exploration of internet marketing strategy by search engine optimization: A critical review and comparison. Afr. J. Bus. Manag. 2011, 5, 4644-4649.

22. Patil Swati, P.; Pawar, B.V.; Patil Ajay, S. Search Engine Optimization: A Study. Res. J. Comput. Inf. Technol. Sci. 2013, 1, 10-13.

23. Hui, Z.; Shigang, Q.; Jinhua, L.; Jianli, C. Study on website Search Engine optimization. In Proceedings of the 2012 International Conference on Computer Science \& Service System (CSSS), Nanjing, China, 11-13 August 2012; pp. 930-933.

24. Gregurec, I.; Grd, P. Search Engine Optimization (SEO): Website analysis of selected faculties in Croatia. In Proceedings of the Central European Conference on Information and Intelligent Systems, Varazdin, Croatia, 19-21 September 2012; pp. 211-218.

25. Chandra, A.; Suaib, M.; Beg, D. Low cost page quality factors to detect web spam. arXiv, 2014; arXiv:1410.2085.

26. Thakur, A.; Sangal, A.L.; Bindra, H. Quantitative measurement and comparison of effects of various search engine optimization parameters on Alexa Traffic Rank. Int. J. Comput. Appl. 2011, 26, 15-23. [CrossRef]

27. Egri, G.; Bayrak, C. The role of search engine optimization on keeping the user on the site. Procedia Comput. Sci. 2014, 36, 335-342. [CrossRef] 
28. Kumar, R.; Saini, S. A study on SEO monitoring system based on corporate website development. Int. J. Comput. Sci. Eng. Inf. Technol. 2011, 1, 42-49.

29. Dean, B. We Analyzed 1 Million Google Search Results. Here's What We Learned about SEO. 2 September 2016. Available online: https:/ / backlinko.com/search-engine-ranking (accessed on 24 January 2018).

30. Fishkin, R. How to Rank in 2018: The SEO Checklist. 2017. Available online: https:// moz.com/blog/rankin-2018 (accessed on 27 December 2018).

31. Palos-Sanchez, P.; Martin-Velicia, F.; Ramon Saura, J. Complexity in the Acceptance of Sustainable Search Engines on the Internet: An Analysis of Unobserved Heterogeneity with FIMIX-PLS. Complexity 2018, 2018, 6561417. [CrossRef]

(C) 2019 by the authors. Licensee MDPI, Basel, Switzerland. This article is an open access article distributed under the terms and conditions of the Creative Commons Attribution (CC BY) license (http:/ / creativecommons.org/licenses/by/4.0/). 